\title{
Charge collection studies in irradiated HV-CMOS particle detectors *
}






\begin{abstract}
:
Charge collection properties of particle detectors made in HV-CMOS technology were investigated before and after irradiation with reactor neutrons. Two different sensor types were designed and processed in 180 and $350 \mathrm{~nm}$ technology by AMS. Edge-TCT and charge collection measurements with electrons from ${ }^{90} \mathrm{Sr}$ source were employed. Diffusion of generated carriers from undepleted substrate contributes significantly to the charge collection before irradiation, while after irradiation the drift contribution prevails as shown by charge measurements at different shaping times. The depleted region at a given bias voltage was found to grow with irradiation in the fluence range of interest for strip detectors at the HL-LHC. This leads to large gains in the measured charge with respect to the one before irradiation. The increase of the depleted region was attributed to removal of effective acceptors removal. The evolution of depleted region with fluence was investigated and modeled. Initial studies show a small effect of short term annealing on charge collection.
\end{abstract}

PACS: 85.30.De; 29.40.Wk; 29.40.Gx

KEYWORDS: HV-CMOS particle detectors, Charge collection, Radiation damage .

*Work performed in the framework of the Strip CMOS collaboration.

${ }^{\dagger}$ Corresponding author; Address: Jožef Stefan Institute, Jamova 39, SI-1000 Ljubljana, Slovenia. Tel: (+386) 1477 3512, fax: (+386) 1477 3166, e-mail: Gregor.Kramberger@ijs.si 


\section{Contents}

1. Introduction 目

2. Samples and experimental technique 2

3. Charge collection measurements 9

3.1 Non-irradiated samples

3.2 Irradiated samples 5

4. Edge-TCT measurements

4.1 Determination of active region

4.2 Active region in irradiated devices 9

4.3 Impact of the weighting field 10

4.4 Annealing effects

5. Effective acceptor removal parameters

6. Predictions of charge collection for different substrate resistivities 13

7. Conclusions

\section{Introduction}

The Large Hadron Collider upgrade [[1, [-] scheduled in the next decade will increase the instantaneous luminosity of proton collisions by an order of magnitude to $5-7 \cdot 10^{34} \mathrm{~cm}^{-2} \mathrm{~s}^{-1}$. In order to harvest the full physics potential of the upgrade a finer segmentation of the tracking detectors is required to cope with the large track density. They should also withstand almost an order of magnitude larger fluence of fast hadrons [3, [- [] without a loss of tracking efficiency. Although the present baseline concept for the upgrade envisages conventional hybrid silicon strip and pixel detectors, which are expected to fulfill the stringent requirements, alternative detector technologies are sought. The possible benefits are: significant accessibility to larger number of manufacturers leading to shorter delivery times, reduced cost, smaller material budget, lower power consumption and better position resolution. The use of CMOS monolithic sensors for particle tracking [5], 6] has been successfully demonstrated over the past two decades in several experiments. The charge collection mechanism in the so-called CMOS-MAPS (monolithic active pixel sensors) relies mainly on diffusion of free carriers generated in the epitaxial silicon layer upon which the electronics resides. The slow diffusion process limits their use at high collision rates. The reduction of carrier recombination lifetimes at larger fluences [ [7] results in significant loss of collected charge, hence their use is restricted to fluences below few times $10^{13} \mathrm{~cm}^{-2}$ [8]. The HV-CMOS process [9] allows 


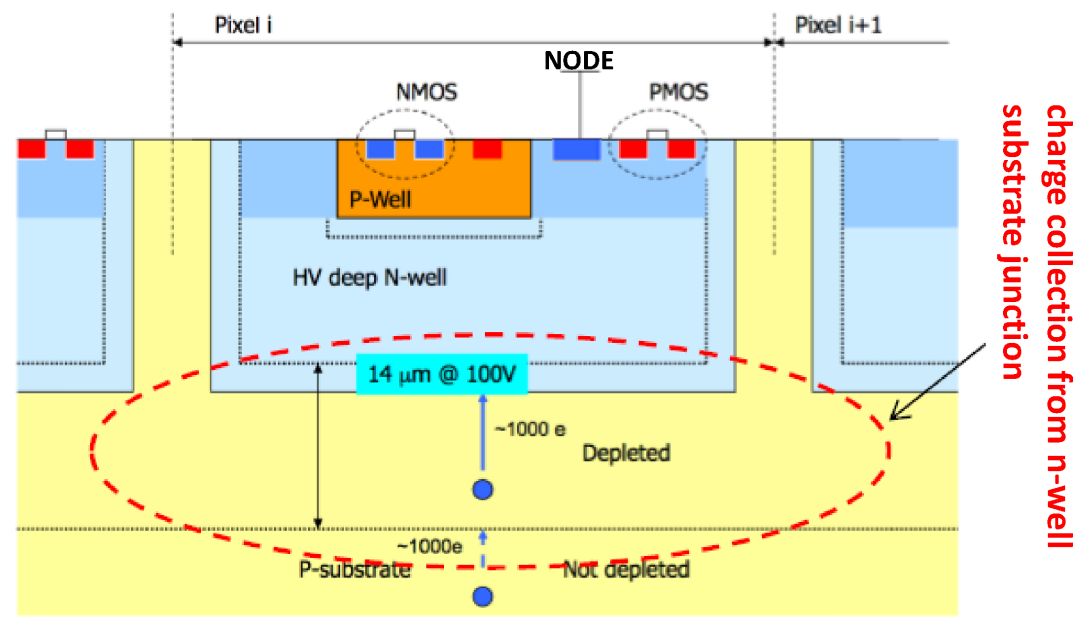

Figure 1. Schematic view of the HV-CMOS detector cross-section. The region of interest, where the depleted region grows, is indicated.

relatively high bias voltage to be applied to the substrate and the deep n-well acts as an electrode implant. The current induced in the readout node is then routed to the in-pixel electronics. The ability to increase the depleted region makes carrier drift the dominant charge collection mechanism at higher bias voltages (see Fig. (1) [10]. In this sense the charge collection of HV-CMOS detectors is similar to the operation of standard silicon particle detectors. Apart from the electronics built in the shallow p-well residing in the deep n-well the most notable difference lies in the much lower substrate resistivity.

The aim of this paper is to determine charge collection properties of the prototype HV-CMOS sensors produced by AMS ${ }^{1}$ after irradiation to high fluences of reactor neutrons. The changes in effective doping concentration were investigated with Edge-TCT and charge collection measurements with minimum ionizing electrons from ${ }^{90} \mathrm{Sr}$ using different shaping times.

\section{Samples and experimental technique}

Two different types of samples were produced by AMS using their high voltage process. The CHESS-1 (Test CHip for CMOS Evaluation of Strip Sensors - 1) sensors were designed within the Strip CMOS collaboration in $350 \mathrm{~nm}$ technology while HV2FEI4 [II] were produced in 180 $\mathrm{nm}$ technology. The two designs are shown in Fig. 2. The substrate resistivities were $20 \Omega \mathrm{cm}$ for CHESS- 1 and $10 \Omega \mathrm{cm}$ for HV2FEI4. Both chips employ several test structures of which only a few were used for this study. Each HV2FEI4 pixel cell comprises a charge sensitive preamplifier whose output is further processed in the readout chain. A single pixel cell of $125 \times 33 \mu \mathrm{m}^{2}$ offers an access to the output of the pre-amplifier and is used in this study for Edge-TCT measurements. At the edge of the CHESS-1 chip there is a $3 \times 3$ matrix of passive diodes/pixels (no signal processing electronics implemented) used for the same purpose. In addition, CHESS-1 chip has a large passive

\footnotetext{
${ }^{1}$ Austria Mikro Systeme A.G., Tobelbader Strasse 30, A-8141 Unterpremstaetten.
} 


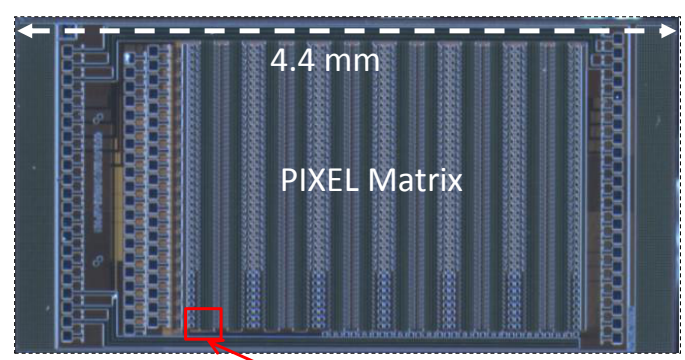

A single cell $125 \times 33 \mu \mathrm{m}^{2}$ with an access to the output of the charge sensitive preamplifier ( $1^{\text {st }}$ amplification stage)

(a)

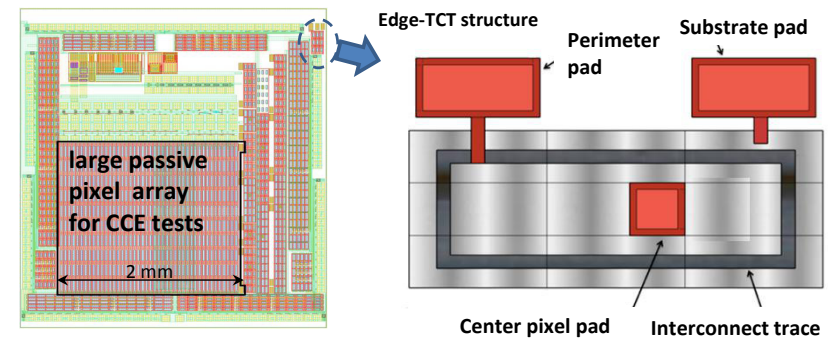

(b)

Figure 2. Layout of the two investigated samples: (a) HV2FEI4 (b) CHESS-1. A single cell from HV2FEI4 in the red square was used for Edge-TCT studies. A large passive diode array in black square of CHESS-1 chip was used for ${ }^{90} \mathrm{Sr}$ measurements and $3 \times 3$ pixel matrix in top left corner for Edge-TCT measurements. Note the scales for (a) and (b) are different and are given in the figures.

diode where 440 pixels of size $200 \times 45 \mu \mathrm{m}^{2}$ were ganged to form a $2 \times 2 \mathrm{~mm}^{2}$ structure used for charge collection efficiency (CCE) measurements with ${ }^{90} \mathrm{Sr}$ electrons. A single HV2FEI4 and three CHESS-1 chips, labeled Dev1, Dev2 and Dev3 were used in this work.

The setup for CCE measurements is shown in Fig. Ba. The electrons from a ${ }^{90} \mathrm{Sr}$ source were collimated by small holes in the top and bottom lid of the Al box housing the sensor and by a hole in the cooled Al support plate. A scintillator matching the sample size was placed below the cold plate underneath the sample. Only close to minimum ionizing electrons could provide sufficiently large signals after crossing the detector in the scintillator-photomultiplier to trigger the readout. The high purity of the trigger (>97\%) enabled measurements even at small signal-to-noise ratios. A large passive array of CHESS-1 samples was connected via a charge sensitive preamplifier to shaping amplifiers with selectable shaping times ranging from 25 to $500 \mathrm{~ns}$. The output of the shaping amplifier was recorded by a digital oscilloscope and stored for offline analysis. The absolute scale of the output signal was carefully calibrated with $59.5 \mathrm{keV}$ photons from ${ }^{241} \mathrm{Am}$ and electrons from ${ }^{90} \mathrm{Sr}$ in a $300 \mu \mathrm{m}$ thick fully depleted standard silicon detector for all shaping times. The details of the setup are described in Ref. [13].



(a)

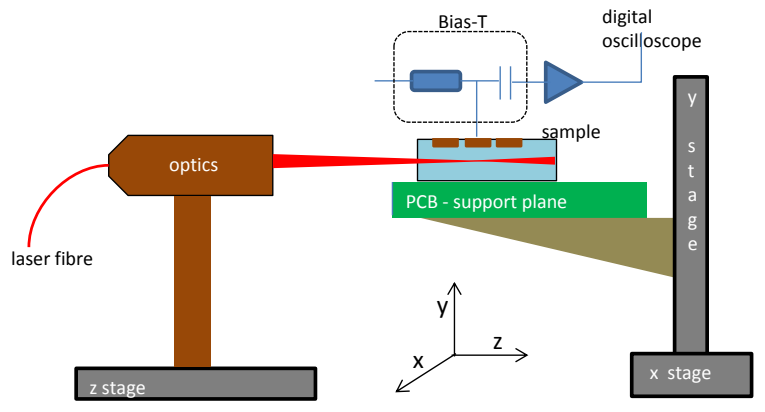

(b)

Figure 3. Schematic view of experimental setups for: (a) charge collection measurements (b) Edge-TCT measurements.

The basic principle of the Edge-TCT is shown in Fig. Bb. The edge of the detector is illu- 
minated with a pulsed narrow laser beam of infrared light $(\lambda=1064 \mathrm{~nm}$, pulse width $\leq 100 \mathrm{ps,}$ $200 \mathrm{~Hz}$ repetition rate, FWHM of beam waist in both directions $\leq 10 \mu \mathrm{m}$ ). Electron-hole pairs are created almost uniformly along the beam in a similar way as for minimum ionizing particles (MIP). The beam position, and by that the depth at which the carriers are generated, is controlled by movable stages with sub-micron precision. The carriers start to drift in the electric field and induce a signal, which is read out by the current amplifier connected to one of the electrodes. An average of around a hundred pulses is recorded by a $1.5 \mathrm{GHz}$ oscilloscope at each scan position. The detailed description of the setup and the measurement technique can be found in Ref. [14]. Ideally, the edge of the detector would be carefully polished to avoid light diffraction in the sample, but due to the small size of the devices under study, this was not possible.

The samples were irradiated at room temperature with neutrons up to the fluence of $1 \cdot 10^{16}$ $\mathrm{cm}^{2}$ at the Jožef Stefan Institute's research reactor [15]. Irradiations were made in steps with short term annealing completed in-between. The detectors were measured with TCT and/or CCE after each irradiation step. In addition to allowing the coverage of many fluence points with limited number of devices the imperfections of the untreated edge of the devices remain the same for all fluences, thus reducing the systematic error of the measurements. The Edge-TCT measurements were performed at room temperature while the CCE measurements were done in the range from $-20^{\circ} \mathrm{C}$ to $20^{\circ} \mathrm{C}$.

\section{Charge collection measurements}

\subsection{Non-irradiated samples}

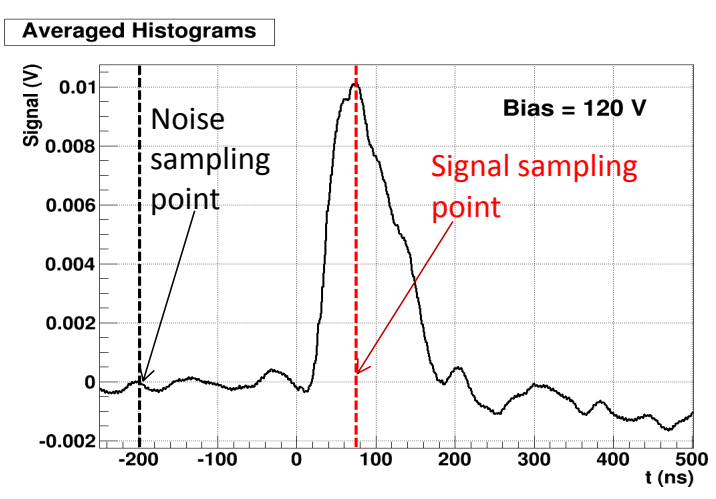

(a)

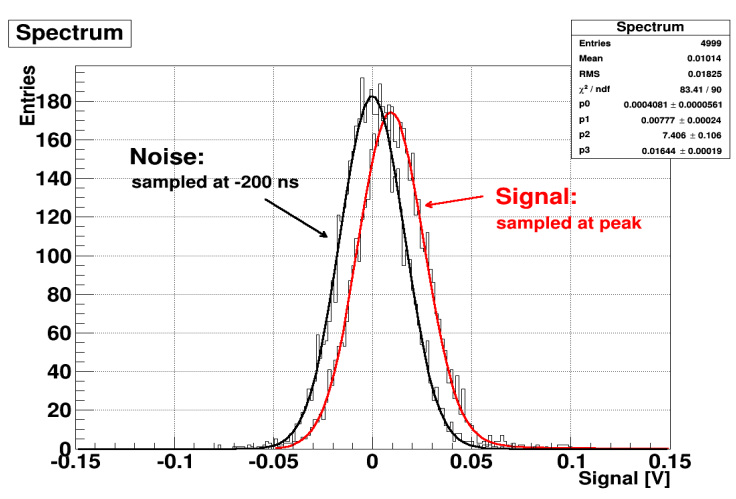

(b)

Figure 4. (a) Output from shaping amplifier, averaged over 5000 events for a substrate bias of 120 V. (b) Spectra of noise and signal. The shift of distribution corresponds to the mean value of charge.

The signal from the shaping amplifier averaged over 5000 events is shown in Fig. Tha for a non-irradiated CHESS-1 passive diode array with substrate biased to $V_{s u b}=120 \mathrm{~V}$. The spectra of signals sampled at the peaking time after the trigger (denoted by the red line in Fig. Ta) and before the trigger (noise sampling - black line) are shown in Fig. Tb. Although they have almost the same shape the signal spectrum is offset by the corresponding mean ionization signal.

A series of CCE measurements were performed with CHESS-1 devices at different shaping times $\tau_{s h}$ (Fig. 5). Good reproducibility of the measured charge, within few hundred electrons 
among all devices can be observed. A strong contribution from carriers collected by diffusion at $V_{s u b}=0 \mathrm{~V}$ is evident at $\tau_{s h}=25 \mathrm{~ns}$ and increases with longer shaping times. Contributions of 1230 e, $2100 \mathrm{e}$ and $2700 \mathrm{e}$ were measured for 25, 200 and $500 \mathrm{~ns}$ shaping times, respectively. Their ratios are roughly consistent with the expectations for the diffusion dominated charge collection [16]. The



Figure 5. Dependence of mean collected charge for CHESS-1 devices on bias voltage for different shaping times.

collected charge increases almost linearly with $V_{s u b}$. The rise can be explained by the depletion of substrate (space charge region) and collection of carriers by drift. As the drift is shorter than $25 \mathrm{~ns}$ there is no ballistic deficit and the drift contribution to the collected charge is independent on the shaping time. The charge collection in an unirradiated device can thus be empirically parameterized by

$$
Q\left(V_{\text {sub }}\right) \approx a+b \cdot V_{\text {sub }},
$$

where $a=a\left(\tau_{s h}\right)$ denotes the diffusion component dependent on shaping time. The drift component exhibits a slope of $b \approx 8.5 \pm 1 \mathrm{e} / \mathrm{V}$. Assuming a mean energy loss of $98 \mathrm{e}-\mathrm{h}$ pairs $/ \mu \mathrm{m}$ by ${ }^{90} \mathrm{Sr}$ electrons triggering the readout [13], the depletion region is around $10 \mu \mathrm{m}$ at $120 \mathrm{~V}$, a value close to the expected one from the initial resistivity of the substrate.

\subsection{Irradiated samples}

Dependence of collected charge on bias voltage for irradiated CHESS-1 detectors is shown in Fig. Ga. It is evident that the diffusion contribution almost vanishes already at the fluence of $\Phi_{e q}=$ $2 \cdot 10^{14} \mathrm{~cm}^{-2}$. At the lowest two fluences, collected charge is smaller than before the irradiation, but for the fluences of $\Phi_{e q}=1-2 \cdot 10^{15} \mathrm{~cm}^{-2}$ it is a factor of two larger. This implies a wider depleted region in the detector than before irradiation. Neutron irradiation introduces deep acceptors [17], which should lead to a reduction of the depleted region. However, it also affects the shallow acceptor (boron) concentration, which decreases with irradiation. Effective initial acceptor removal has also been observed in different detectors with high boron concentration [18, [19, 20]. As the acceptors are removed at higher rates than deep acceptors are created the effective net concentration of negative space charge initially decreases. 


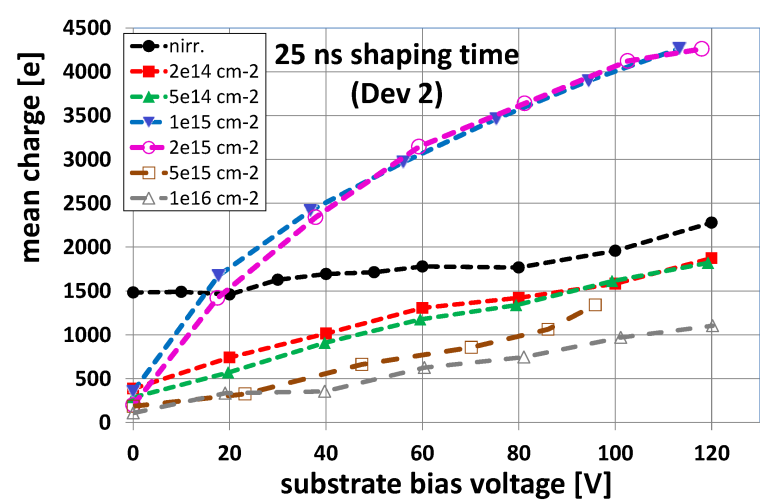

(a)

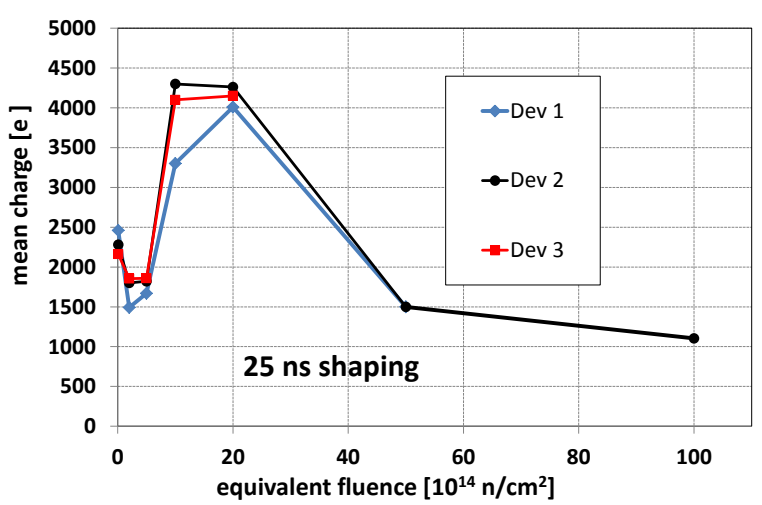

(b)

Figure 6. (a) Dependence of mean charge of CHESS-1 devices on bias voltage at different fluences. (b) Mean charge at $120 \mathrm{~V}$ vs. fluence for different devices. The measurements shown at $5 \cdot 10^{15} \mathrm{~cm}^{-2}$ were taken at $100 \mathrm{~V}$, due to onset of micro-discharges. The lines are used to guide the eye.

At very high fluences the mean collected charge decreases again, due to increase of the deep acceptor concentration and trapping probability of the drifting carriers [21]. The dependence of mean collected charge at the bias of $120 \mathrm{~V}$ on $1 \mathrm{MeV}$ neutron equivalent fluence is shown in Fig. 6b. A good reproducibility of CCE measurement for different devices can be observed. Even after the highest fluence of $10^{16} \mathrm{~cm}^{-2}$, the collected charge degrades moderately when compared to standard detectors considering only the drift component.

At $\Phi_{e q}=1-2 \cdot 10^{15} \mathrm{~cm}^{-2}$ sizeable depletion depths can be achieved at the applied voltages. Therefore the growth of depleted region as $\sqrt{V_{s u b}}$ for homogeneous $N_{\text {eff }}$ becomes more pronounced and the effective linear approximation Eq. 3.1 is not adequate.

\section{Edge-TCT measurements}

The conclusions made in the section on charge collection measurements were verified by EdgeTCT method [14], which is an ideal tool to study substrate properties. Typical response waveforms taken during TCT measurements are shown in Figs. Z7a,b. As already mentioned the waveform from HV2FEI4 (Fig. 7a) corresponds to measured charge $\left(Q(t) \propto \int_{0}^{t} I\left(t^{\prime}\right) d t^{\prime}\right)$, while for CHESS-1 it (Fig. Zb) is the induced current pulse convoluted with the transfer function of the system. The contribution from the diffusion can be observed for the HV2FEI4 chip, where measured charge for injection deep in the sensor $(y=35 \mu \mathrm{m})$ and close to the surface $(y=95 \mu \mathrm{m})$ is about the same, but for different reasons. Illumination of the top side results in faster signal, reaching the maximum in around $50 \mathrm{~ns}$, but only a fraction of the light entered the detector. At larger depths the beam is contained in the sensor, but the carriers also recombine in low field and it takes considerably longer to reach maximum signal. The measurements of the induced currents in CHESS-1 lead to the same conclusion. Even though the measured induced charge at $50 \mu \mathrm{m}$ and $70 \mu \mathrm{m}$ (close to the surface) are similar, there is a huge difference in the fast component in the first few ns.

The corresponding charge profiles at $60 \mathrm{~V}$ are shown in Figs. Z7 c,d. The charge sampled at 500 ns is assumed for HV2FEI4 while current integral over $25 \mathrm{~ns}$ is taken for CHESS-1. In both cases the sensitive area of the pixel in the $x-y$ plane is well reproduced. It can be clearly seen that the 
high charge collection region is around $20 \mu \mathrm{m}$ wide, but the contribution comes also from depths of several ten $\mu \mathrm{m}$.

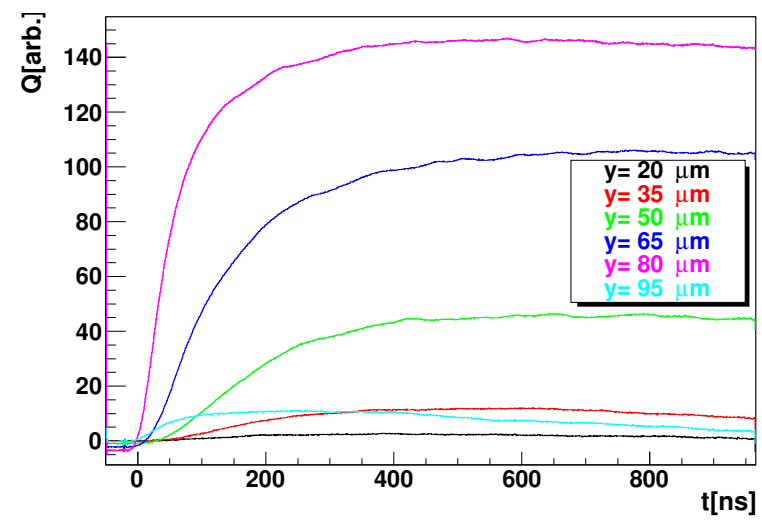

(a)



(c)

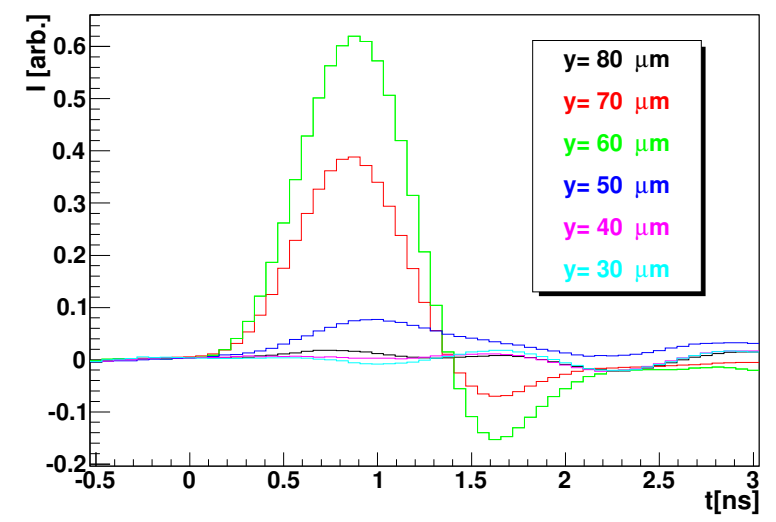

(b)

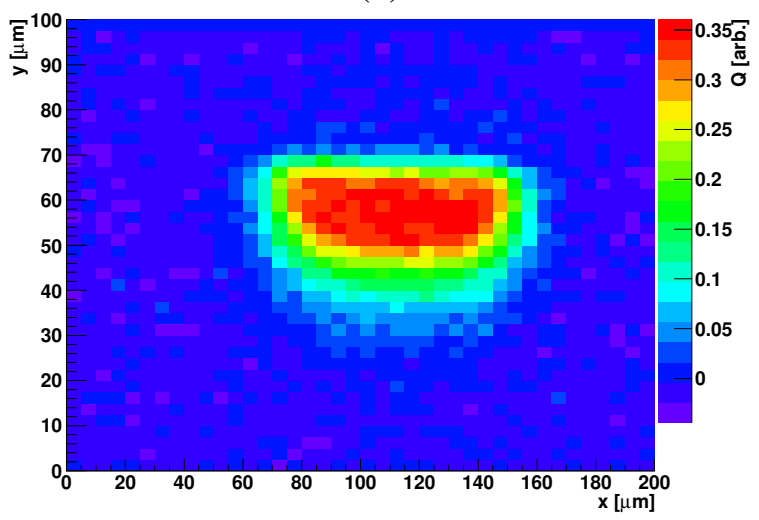

(d)

Figure 7. Measured waveforms from a non-irradiated (a) HV2FEI4 chip and (b) CHESS-1 after IR laser pulse illumination for different depths through the centre of the chip in $x$ at $60 \mathrm{~V}$. The collected charge at different $x$-width and $y$-depth at $60 \mathrm{~V}$ for: (c) HV2FEI4, sampled at $500 \mathrm{~ns}$ and (d) CHESS-1 integrated for $25 \mathrm{~ns}$. All measurements were performed at room temperature.

\subsection{Determination of active region}

The charge collection profile along the centre of the pixel is shown in Figs. 8 $8 \mathrm{a}, \mathrm{b}$. The surface of the chip is at $y \approx 90 \mu \mathrm{m}$ for HV2FEI4 and $y \approx 70 \mu \mathrm{m}$ for CHESS- 1 . The rise of the charge as the beam enters the detector to its maximum occurs in around 8-10 $\mu \mathrm{m}$, which agrees with the focusing performance of the optics. The maximum charge is almost independent of bias voltage which is expected when all generated free carriers are collected, by either drift or diffusion. The decrease of the charge as the beam moves deeper in the sensor can be attributed to less charge collected by diffusion, which takes longer than the investigated collection times. This leads to an asymmetric profile. CHESS-1 chip could be biased to larger voltages and had a smaller initial doping concentration. As the beam width was smaller than the depleted region a flat top in $Q(y)$ clearly corresponds to creation of all charge in the depleted region. 
With Edge-TCT the local electric field can be probed by looking at the induced current promptly (600 ps) after carrier injection [14]. The measured current is in this case proportional to the sum of drift velocities at a given depth $\left(v_{e}(y)+v_{h}(y)\right)$. The velocity profile for a passive pixel of CHESS-1 is shown in Fig. $8 \mathrm{c}$. The maximum velocity increases with applied bias and there is no tail at smaller $y$. The point where $v_{e}+v_{h} \sim 0$ therefore indicates the border of the depleted region. The falling and rising edges are compatible with the beam width which means two things: the electric field is already high a few $\mu \mathrm{m}$ away from the edge and the full width at half maximum (FWHM) of the velocity profile corresponds approximately to the width of the depleted region. As an illustration, the width of the profile increases by $8 \mu \mathrm{m}$ when the bias voltage increases from 20 to $100 \mathrm{~V}$, which corresponds well to the difference of around 750 e observed in the charge with ${ }^{90} \mathrm{Sr}$.



(a)

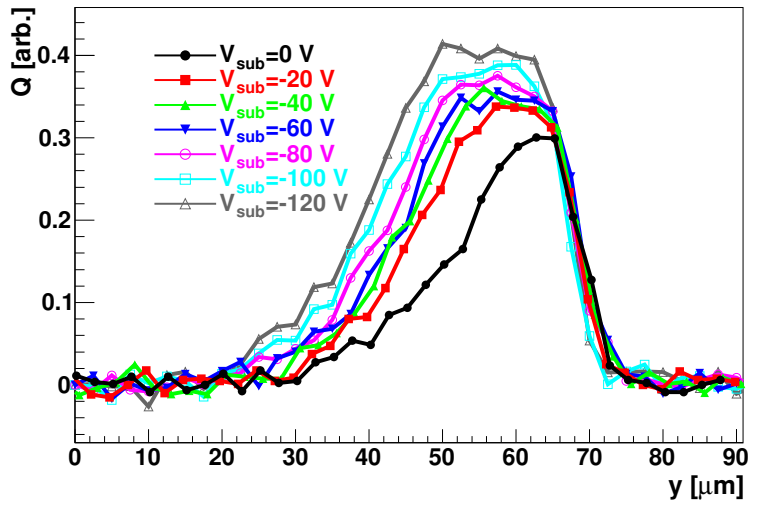

(b)

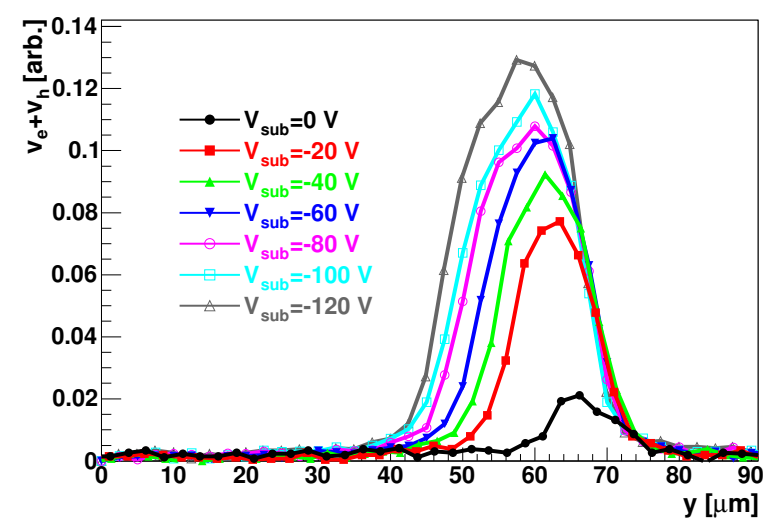

(c)

Figure 8. Charge collection profile for: (a) HV2FEI4, (b) CHESS-1 and (c) velocity profile for CHESS-1, all through the centre of the pixel. Measurements were performed at room temperature.

The increase of the depleted region (high field region) can be also evaluated by comparing the FWHM of the charge collection profiles. Under the assumption of additive contributions from drift and diffusion an increase of charge collection profile width can be attributed to the extension of the depleted region. As both methods agree well (see Figs. $8 \mathrm{~b}, \mathrm{c}$ ) and only the latter can be applied to both HV2FEI4 and CHESS-1, it will be further used for evaluation of the depth of the depleted region. 


\subsection{Active region in irradiated devices}

The charge collection profiles for irradiated devices are shown in Fig. 9. The increase of depleted region depth is clearly visible when compared to the one before the irradiation thus confirming the measurements with ${ }^{90} \mathrm{Sr}$. Vanishing contribution of diffusion to the measured charge is evident at 0 $\mathrm{V}$, where almost no charge is detected, due to the large reduction of minority carrier lifetime with irradiation [[]].
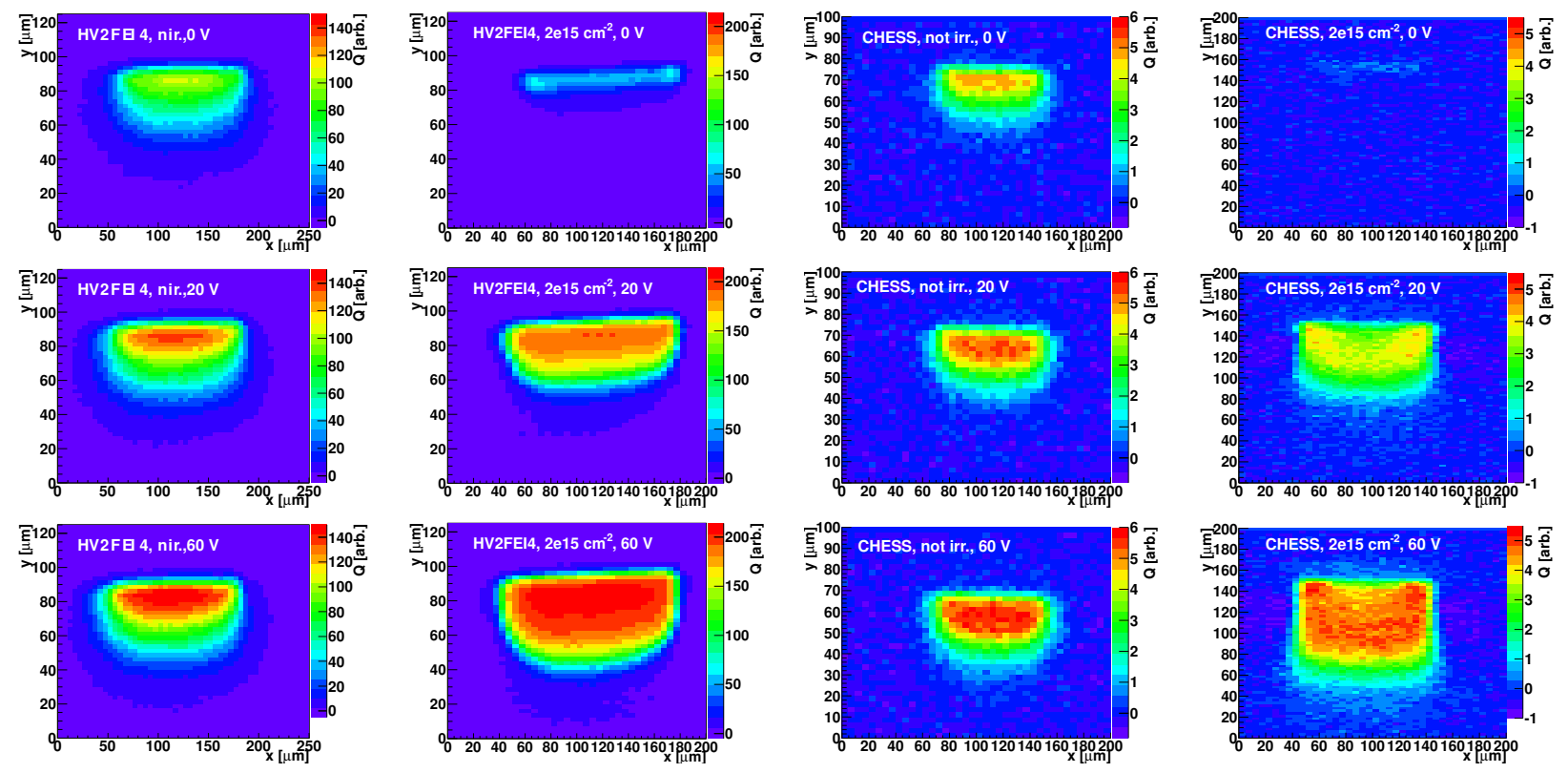

Figure 9. The charge profiles at different substrate bias voltages for HV2FEI4 and CHESS-1 before and after irradiation to the fluence of $\Phi_{e q}=2 \cdot 10^{15} \mathrm{~cm}^{-2}$. Measurements were performed at room temperature. Note the different $x$ and $y$ axis ranges.

The charge collection profiles measured along the centre of the pixel for HV2FEI4 are shown in Fig. 10a. The width increases with bias voltage, while the diffusion tail disappears, hence the falling and rising edge of the profiles are the same. The change of charge collection width at 60 $\mathrm{V}$ for different fluences is shown in Fig. [10b. After initial decrease, due to loss of diffusion, the profile width starts to increase and reaches the maximum around $1-2 \cdot 10^{15} \mathrm{~cm}^{-2}$ and then starts to decrease. Such a behavior is in agreement with charge collection measurements.

The dependence of the charge collection profile FWHM on bias voltage for different fluences is shown in Fig. [1]. For both detector types the evolution of the charge profile width is similar. If the constant space charge $\left(N_{e f f}\right)$ is assumed in the space charge region, then the width should increase with the substrate voltage as

$$
d=d_{0}+\sqrt{\frac{2 \varepsilon_{S i}}{e_{0} N_{e f f}} V_{s u b}},
$$

where $d_{0}$ is the width at $V_{s u b}=0 \mathrm{~V}, \varepsilon_{S i}$ the permittivity of Silicon and the $e_{0}$ elementary charge. Eq. 4.1 (shown in Figs. 111) fits the data well, therefore allowing the determination of the corresponding constant effective doping concentration. Although the measured $N_{\text {eff }}$ obtained from the fit roughly 


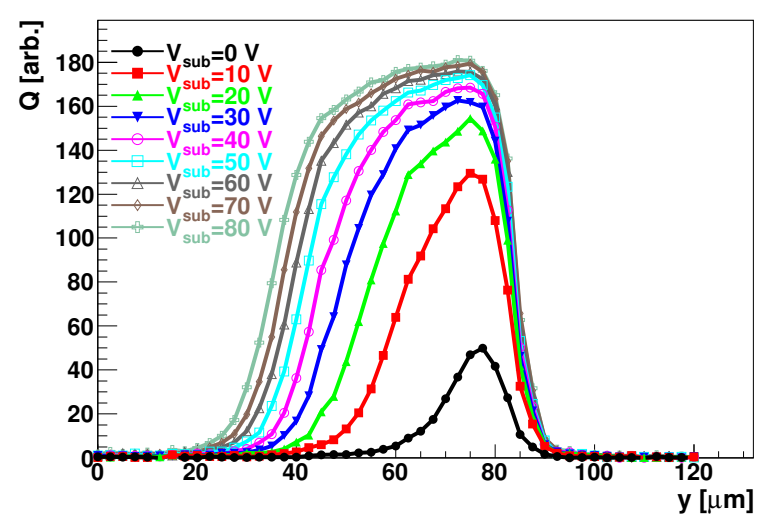

(a)

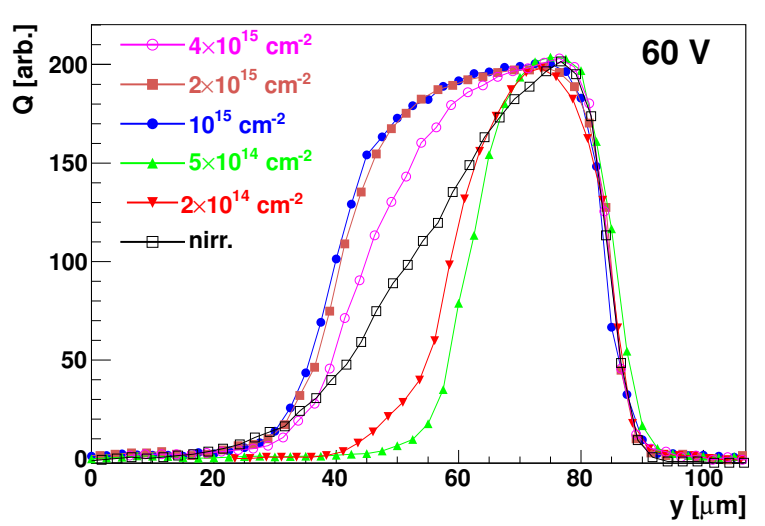

(b)

Figure 10. Charge collection for HV2FEI4 detector (a) after $\Phi_{e q}=1 \cdot 10^{15} \mathrm{~cm}^{-2}$ for different bias voltages and (b) at $60 \mathrm{~V}$ for different fluences. Profiles in (b) are normalized to the maximum point.



(a)

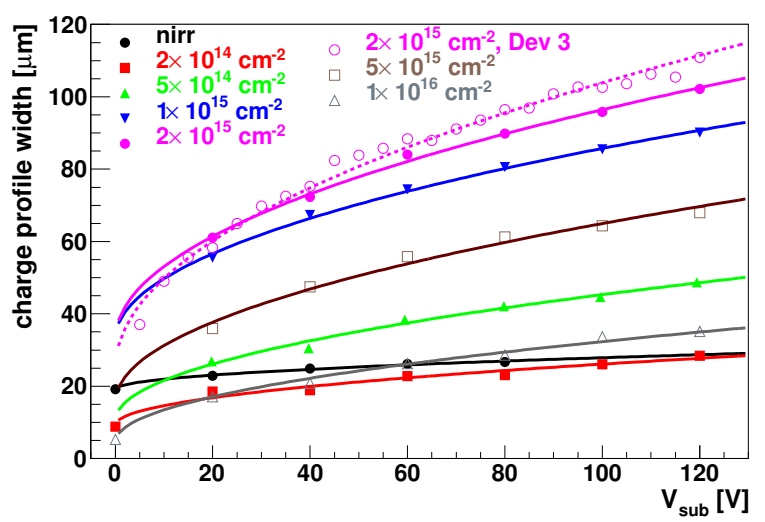

(b)

Figure 11. Dependence of charge profile width on bias voltage at different fluences for (a) HV2FEI4 and (b) CHESS-1, Dev 2. The fits of Eq. (4.1) to the measured data are shown.

agree for both devices at large fluences (see Section 5), the measured widths for CHESS-1 were larger than those for HV2FEI4. For example at $2 \cdot 10^{15} \mathrm{~cm}^{-2}$, where the difference is the largest, the difference is around $30 \mu \mathrm{m}$ at $60 \mathrm{~V}$. The $d_{0}$ obtained from the fit agrees well with the measured one for HV2FEI4 and corresponds to the width of the laser beam. This is not the case with CHESS-1, where the measured beam width and $d_{0}$ disagree in the mid-fluence range. A measurement with another device (Dev 3) was performed with a finer step of $5 \mathrm{~V}$ in order to investigate the depletion depth at low voltages and also test the reproducibility among different devices. Again the width increases rapidly at bias voltages of a few $\mathrm{V}$, pointing to the depletion of a region with small space charge, and then rises in accordance with constant $N_{\text {eff }}$.

\subsection{Impact of the weighting field}

The charge collection and velocity profiles along the pixel center after irradiation to $\Phi_{e q}=2 \cdot 10^{15}$ $\mathrm{cm}^{-2}$ are shown in Figs. 12a,b for CHESS-1. Unlike for HV2FEI4 (see Fig. 10a) the collected charge strongly depends on voltage for beam positions close to the surface. This can be partially 
attributed to the trapping of the drifting charge, but largely it is a sign that carriers don't drift over the entire weighting potential [22, 23]. A possible explanation would be that the effective position of the second electrode changes after irradiation as the resistivity of undepleted silicon increases and it is not at the border of the depleted region anymore [24], hence irradiation provokes an effective change of electrode configuration. An evidence of strongly peaked weighting field is reflected in the position of the maximum in charge collection profile at a given voltage. It is at the edge of the depleted region (see Fig. 12a). Injecting the carriers there leaves the electrons to drift over the entire thickness. Although the electrons start their drift in the low field region (see Fig. [12b), they induce more charge due to smaller trapping probability than the holes which dominate the induced charge for injection close to the surface (illumination at $y \sim 160 \mu \mathrm{m}$ in Fig. 12a).

Moreover, the charge profile exhibits a tail, much wider than the width of the beam. It starts at the point where the velocity drops and is an indication of a substantial low field region.

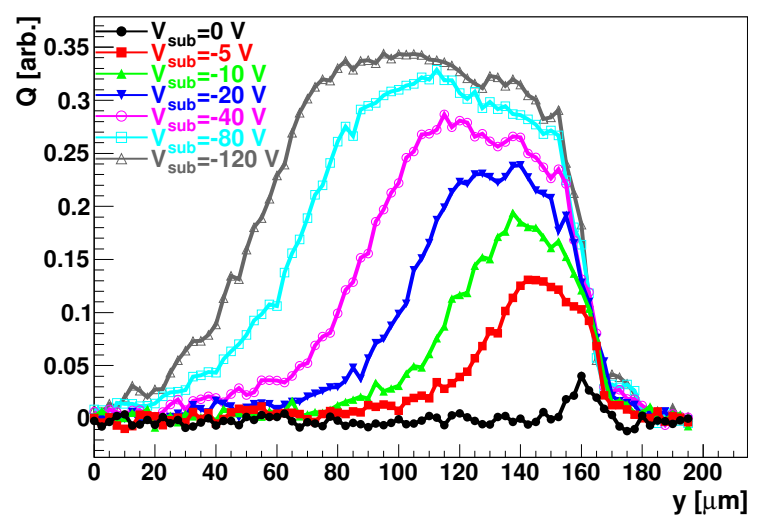

(a)

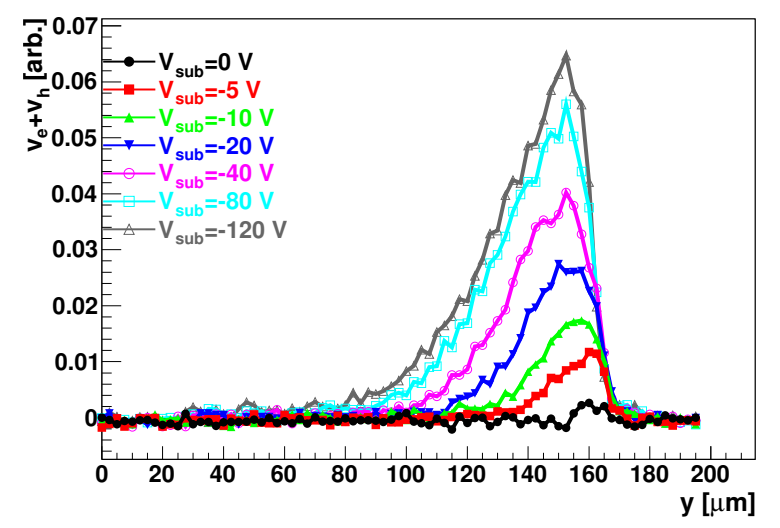

(b)

Figure 12. (a) Charge and (b) velocity profiles along the pixel centre at different bias voltages for a CHESS1Dev2 irradiated to $2 \cdot 10^{15} \mathrm{~cm}^{-2}$.

TCT measurements shown up to now had the centre pixel of CHESS-1 (see Fig. 2 b connected to the readout while the periphery was grounded. A more uniform weighting field is achieved by ganging and reading out all 9 pixels thereby reducing the so called small electrode effect [25]. The difference in induced current is evident in Fig. 13a. While the induced current for the single pixel configuration exhibits a larger increase when electrons reach the electrode than the ganged pixels, the latter exhibit longer tails. As a consequence the charge collection profiles look different, as shown in Fig. [13b. It is clear that for ganged pixel configuration the maximum charge is obtained for injections close to the electrode. The difference is smaller for low fluences where trapping is not so pronounced and at very high fluences where the mean drift distance becomes smaller than the depleted region.

\subsection{Annealing effects}

Irradiation in steps allowed only for exploring the effects of short term annealing. The annealing for $80 \mathrm{~min}$ at $60^{\circ} \mathrm{C}$ after each irradiation step is chosen as the standard procedure, the so called CERN scenario [17], for high resistivity silicon. However, it has not been explored for low resistivity substrates. Usually the measurements were also performed before any intentional annealing. 


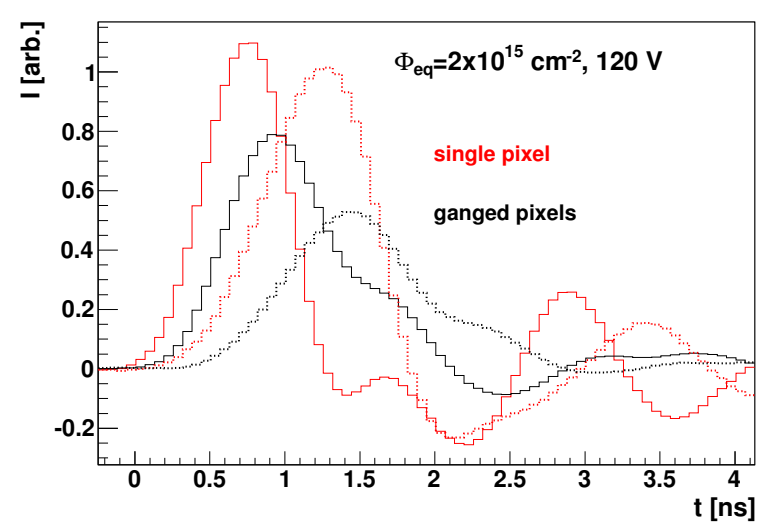

(a)

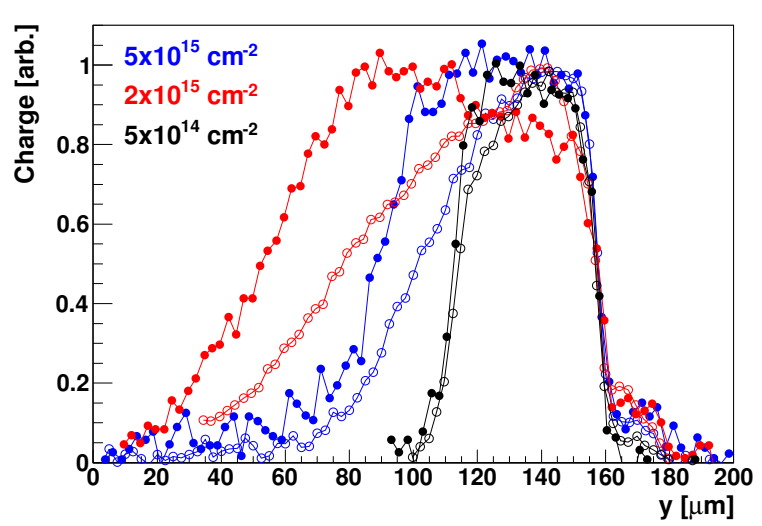

(b)

Figure 13. (a) Comparison of induced currents in the single and ganged pixel configuration for carriers injected close to the surface (solid line) and $50 \mu \mathrm{m}$ away from it (dotted line).(b) Charge collection profiles for single pixel (solid markers) and ganged pixel (open markers) configuration at $V_{s u b}=120 \mathrm{~V}$. The profiles are normalized to the maximum point.

Unlike for standard silicon detectors where an improvement in charge collection efficiency of order 20-30\% is observed after annealing, short term annealing has no impact on CCE of CHESS-1 detectors (see Fig. 14a). The charge collection profile width for HV2FEI4 also shows very little, if any, change due to short term annealing (see Fig. 14b).

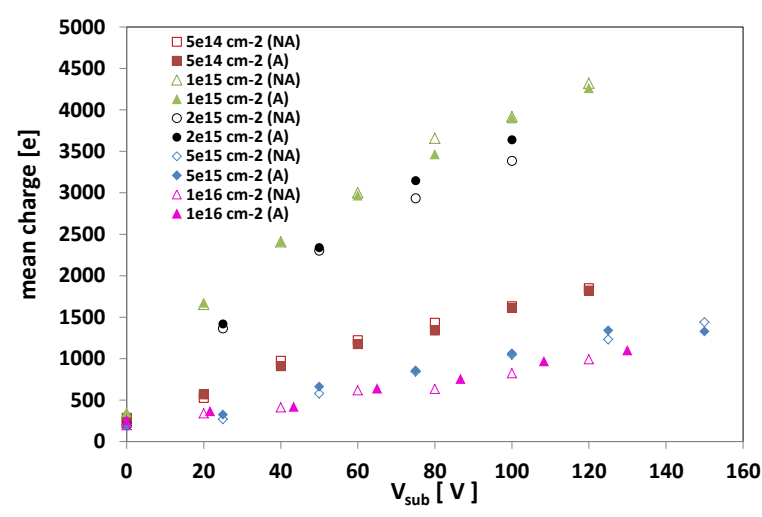

(a)

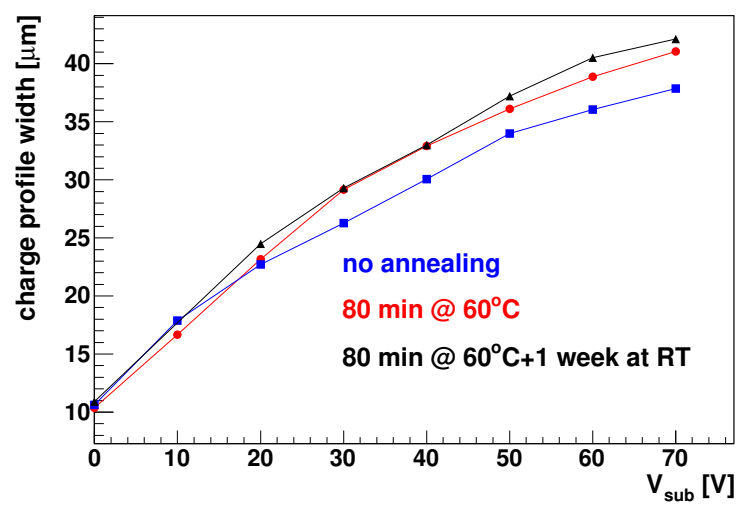

(b)

Figure 14. (a) Dependence of mean collected charge on voltage for different fluences before short term annealing (NA, open markers) and after it (A, solid markers). (b) Comparison of charge profile width for HV2FEI4 after accumulated fluence of $\Phi_{e q}=4 \cdot 10^{15} \mathrm{~cm}^{-2}$ at different annealing stages.

\section{Effective acceptor removal parameters}

The removal of effective initial acceptors leads to large improvement of the device performance in terms of charge collection. The removal rate plays an important role in choosing the initial resistivity of the material. The effective doping concentration as extracted from the fit of Eq. 4.1] to the data (Fig. 11) is shown in Fig. 15. The measured points were fit by the equation, describing 


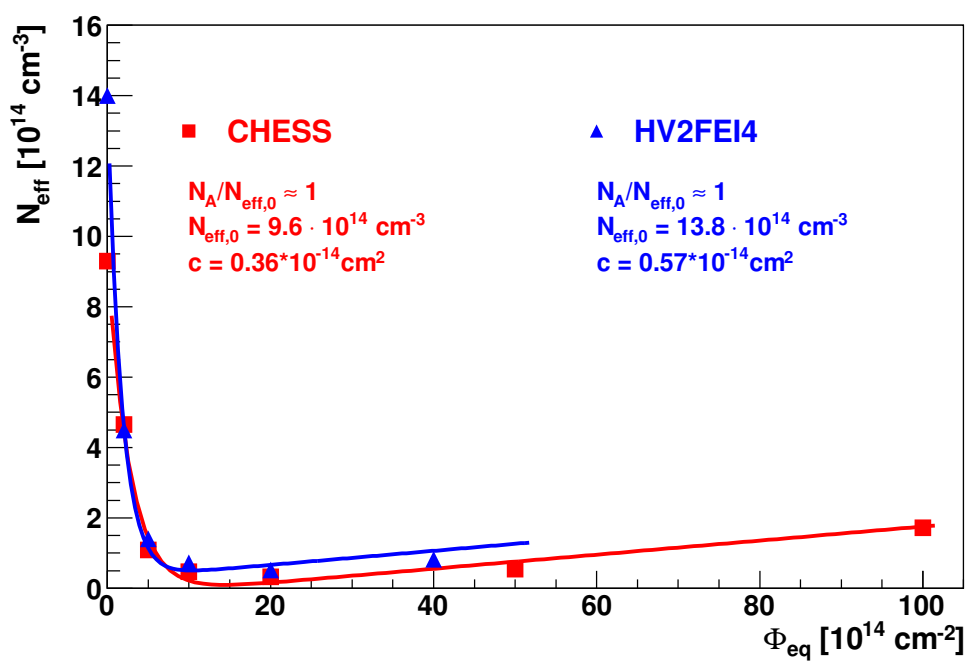

Figure 15. Dependence of substrate's effective doping concentration on irradiation fluence for both chips. The fit parameters obtained from the fit of Eq. (5.1) to the measured data are also shown.

exponential removal of acceptors and generation of deep stable acceptors. It was assumed that the short term annealing of $N_{\text {eff }}$ is completed, while the long term annealing only takes place on much longer time scales, thus:

$$
N_{e f f}=N_{e f f, 0}-N_{A}\left(1-\exp \left(-c \cdot \Phi_{e q}\right)\right)+g_{C} \Phi_{e q}
$$

where $N_{e f f, 0}$ is the initial acceptor concentration of the substrate, $N_{A}$ the concentration of the removed acceptors, $c$ the removal constant and $g_{c}=0.02 \mathrm{~cm}^{-1}$ the generation rate of stable deep acceptors [17]. The free parameters of the fit were $N_{e f f, 0}, N_{A} / N_{e f f, 0}$ and $c$ and their values can be found in Fig. 15. The acceptor removal seems to be complete, i.e. $N_{A}=N_{\text {eff }, 0}$ while $c=0.57 \cdot 10^{-14} \mathrm{~cm}^{2}$ and $c=0.36 \cdot 10^{-14} \mathrm{~cm}^{2}$ were found for HV2FEI4 and CHESS-1 respectively. The uncertainty of these values was around $30 \%$ and depends mostly on the pre-irradiation $N_{\text {eff }}$. The values of the removal constant are 2-3 orders of magnitude smaller than measured for high resistivity silicon [18], but larger than for the removal of acceptors in more doped amplification layer of Low Gain Avalanche Detectors [19].

\section{Predictions of charge collection for different substrate resistivities}

The prediction of collected charge for MIP-s is difficult to derive from Edge-TCT measurements. The generated charge can be determined from the width of the charge collection profile assuming the mean energy loss for ${ }^{90} \mathrm{Sr}$ electrons triggering the readout, but for an irradiated detector the trapping and geometry effects (weighting field, inter-pixel regions with lower efficiency) should also be accounted for.

A simple simulation however enables a rough estimate of the collected charge for a large pad of HV-CMOS detectors of different substrate resistivities. The details of simulation can be found in Ref. [26]. The simulator solves the Poisson equation for a given effective doping concentration 


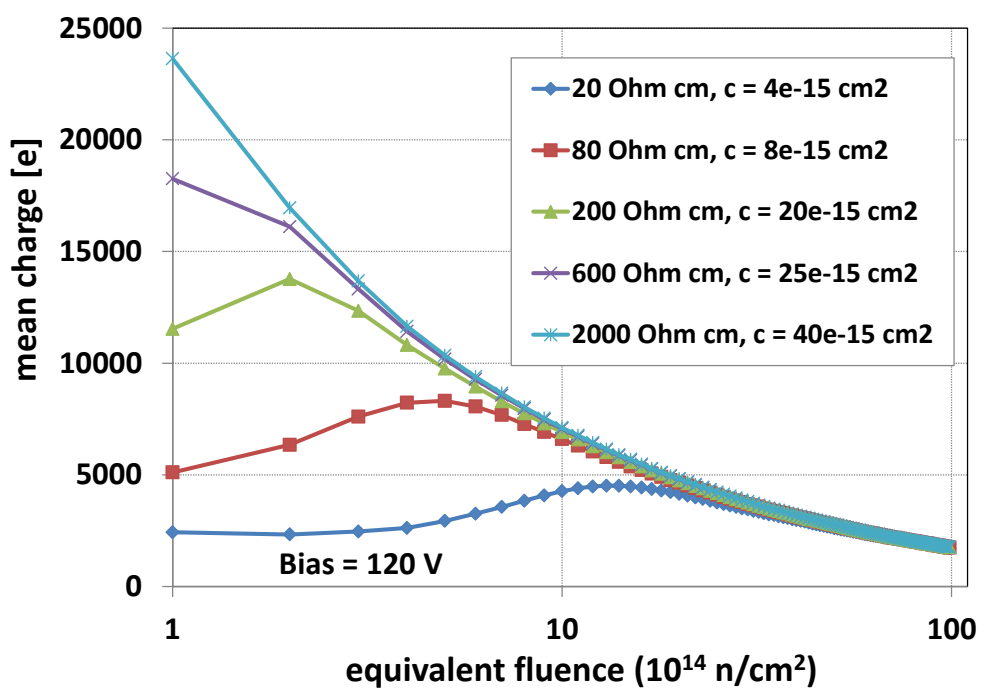

Figure 16. Dependence of simulated mean charge on equivalent neutron fluence in a large HV-CMOS pad detector for different substrate resistivities.

to obtain the electric field profile. The drift of the generated charge is done in steps and their contribution to the induced current calculated taking into account trapping time constants from [21] and mobility parametrization from [27]. The effective doping concentration was calculated from Eq. 5.1. However, the removal constant $c$ depends on the initial doping concentration, which was only roughly approximated for different initial resistivities from [28]. As can be seen in Fig. 16 higher resistivity is beneficial below $\Phi_{e q}<2 \cdot 10^{15} \mathrm{~cm}^{-2}$. Above that fluence the initial resistivity is not important, while below the fluence of maximum charge collection is a function of resistivity. Ideally a substrate resistivity can be selected for which the CCE doesn't degrade at all in the fluence range of interest. Therefore the use of HV-CMOS detectors in terms of charge collection/detection efficiency offers a superb radiation hardness for outer layers of the tracker.

\section{Conclusions}

The charge collection studies on HV-CMOS particle detectors was investigated by Edge-TCT and charge collection measurements with ${ }^{90} \mathrm{Sr}$ electrons. Both complementary techniques agreed with each other. It was demonstrated that the diffusion component disappears at relatively low fluences, but the depleted region increases due to effective removal of initial acceptors. For the investigated devices the collected charge therefore increases with irradiation to few times of that before irradiation and reaches its maximum around $1-2 \cdot 10^{15} \mathrm{~cm}^{-2}$. At larger fluences deep acceptor generation and trapping reduce CCE, but after $10^{16} \mathrm{~cm}^{-2}$ it still remains on the same level as before irradiation. The short term annealing seems to have no impact on charge collection.

\section{References}

[1] F. Gianotti, M.L. Mangano, T. Virdee et al., "Physics potential and experimental challenges of the LHC luminosity upgrade" hep-ph/0204087, 2002. 
[2] P. Allport, M. Nessi, "Letter of Intent for the Phase-II Upgrade of the ATLAS Experiment", CERN-LHCC-2012-022; LHCC-I-023.

[3] I. Dawson on behalf of ATLAS collaboration, "Radiation background simulation and verification at the LHC: Examples from the ATLAS experiment and its upgrades", Proceedings of Science, 015, 2012.

[4] I. Dawson on behalf of ATLAS collaboration, "Radiation background studies for the Phase II inner tracker upgrade", ATL-UPGRADE-PUB-2014-003, 2014.

[5] R. Turchetta, J.D. Berst, B. Casadei et al., "A monolithic active pixel sensor for charged particle tracking and imaging using standard VLSI CMOS technology", Nucl. Instr. and Meth. A 458 (2001) p. 667.

[6] G. Deptuch, J.D. Berst, G. Claus et al. "Design and testing of Monolithic Active Pixel Sensors for charged particle tracking", IEEE Trans. Nucl. Sci. 49(2) (2002) p. 601.

[7] B. L. Gregory, "Minority carrier recombination in neutron irradiated silicon", IEEE Trans. Nucl. Sci. 16(6) (1969) p. 53.

[8] M. Deveaux, G. Claus, G. Deptuch et al., "Neutron radiation hardness of monolithic active pixel sensors for charged particle tracking", Nucl. Instr. and Meth. A512 (2003) p. 71.

[9] R. Minixhofer, N. Feilchenfeld, M. Knaipp et al., "A 120V 180nm High Voltage CMOS smart power technology for System-on-chip integration", Proceedings of the International Symposium on Power Semiconductor Devices \& ICs (2010) p. 75.

[10] I. Perić, "A novel monolithic pixelated particle detector implemented in high-voltage CMOS technology”, Nucl. Instr. and Meth. A582 (2007) p. 876.

[11] I. Perić, "Active pixel sensors in high-voltage CMOS technologies for ATLAS", J. of Instrumentation Vol. 7 (2012) C08002.

[12] I. Perić, R. Eber, F. Ehrler et al., "Overview of HVCMOS pixel sensors”, J. of Instrumentation Vol. 10 (2015) C05021.

[13] G. Kramberger, V. Cindro, I. Mandić et al., "Charge collection properties of heavily irradiated epitaxial silicon detectors", Nucl. Instr. and Meth. A554 (2005) 212.

[14] G. Kramberger, V. Cindro, I. Mandić et al.,'Investigation of Irradiated Silicon Detectors by Edge-TCT “, IEEE Trans. Nucl. Sci. Vol. 57(4), 2010, p. 2294.

[15] L. Snoj, G. Žerovnik, A. Trkov, "Computational analysis of irradiation facilities at the JSI TRIGA reactor”, Appl. Radiat. Isot. 70 (2012) p. 483.

[16] L. D. Edmonds, "A Time-Dependent Charge-Collection Efficiency for Diffusion”, IEEE Trans. Nucl. Sci. 48(5) (2001) p. 1609.

[17] G. Lindström on behalf of CERN-RD48 collaboration, "Radiation hard silicon detectors developments by the RD48 (ROSE) collaboration”, Nucl. Instr. and Meth. A 466 (2001) p. 308.

[18] R. Wunstorf, W.M. Bugg, J. Walter et al. "Investigations of donor and acceptor removal and long term annealing in silicon with different boron/phosphorus ratios", Nucl. Instr. and Meth. A377 (1996) 228.

[19] G. Kramberger, M. Baselga, V. Cindro et al.,"Radiation effects in Low Gain Avalanche Detectors after hadron irradiations", J. of Instrumentation Vol. 10 (2015) P07006.

[20] H.L. Snoek on behalf of LHCB VELO group, "The LHCb VELO : Performance and radiation damage", Nucl. Instr. and Meth. A 765 (2014) 35. 
[21] G. Kramberger, V. Cindro, I. Mandić et al., "Effective trapping time of electrons and holes in different silicon materials irradiated with neutrons, protons and pions”, Nucl. Instr. and Meth. A481 (2002) 297.

[22] S. Ramo, “Currents Induced by Electron Motion”, Proceedings of I.R.E. 27 (1939) p. 584.

[23] E. Gatti, G. Padovini, V. Radeka, "Signal evaluation in multielectrode radiation detectors by means fo a time dependent weighting vector”, Nucl. Instr and Meth. 193 (1982) 651.

[24] Z. Li, "Modeling and simulation of neutron induced changes and temperature annealing of $N_{\text {eff }}$ and changes in resistivity in high resistivity silicon detectors", Nucl. Instr. and Meth. A 342 (1994) 105.

[25] J. D. Eskin, H. H. Barrett and H. B. Barber, "Signals induced in semiconductor gamma-ray imaging detectors", J. Appl. Phys. 85 (1999) p 647.

[26] G. Kramberger, V. Cindro, I. Mandić et al., "Influence of trapping on silicon microstrip detector design and performance”, IEEE Trans. Nucl. Sci. Vol. 49(4) (2010) p. 1717.

[27] C. Canali, G. Ottavian, A. Alberigi, "Drift velocity of electrons and holes and associated anisotropic effects in silicon”, J. of Phys. Chem. Solids, vol. 32 (1971) p. 1707.

[28] G. Kramberger, V. Cindro, I. Mandić et al., "Initial acceptor removal in p-type silicon detectors", Presented at $10^{\text {th }}$ Trento Workshop on advanced silicon radiation detectors (3D and p-type), Trento, February 2015. 